\title{
Las nuevas narrativas en el proceso de enseñanza- aprendizaje en las facultades públicas de Periodismo
}

\section{Berta García Orosa ${ }^{a}$ y Lara Lozano Aguiar ${ }^{b}$}

aProfesora Titular en la Facultad de Ciencias de la Comunicación y miembro del grupo de investigación Novos Medios de la USC (berta.garcia@usc.es), bDoctoranda y colaboradora del grupo de investigación Novos Medios de la USC (laralozanoaguiar@gmail.com).

\begin{abstract}
The emergence of new social media platforms has produced a significant change, not only in academic content in university course of studies, but also in the presentation of these to students. The teaching-learning processes are currently marked by the demands of the new narratives. But... What extent have the contents of the curriculum of the degrees in Journalism taught in the public universities in Spain? In order to answer this question, we have analyzed the way in which the Spanish public schools present the offers of Degree in Journalism through the websides. Furthermore, we have deepened in the characteristics of the curriculum of those subjects whose content be about the demands of digital narratives such as multimedia, transmedia and hypertext.
\end{abstract}

Keywords: new narratives, transmedia, management communication, journalism and university teaching processes

\begin{abstract}
Resumen
La irrupción de las nuevas plataformas de social media han producido un cambio significativo, no solo en los contenidos académicos en los planes de estudios universitarios, sino también a la hora de presentar éstos a los alumnos. Los procesos de enseñanza-aprendizaje se ven marcados actualmente por las exigencias de las nuevas narrativas, pero ihasta que punto se han actualizado y adecuado a la era 2.0 los contenidos de los planes de estudios de los grados en Periodismo impartidos en las universidades públicas en España?

Con el fin de dar respuesta a esta cuestión, hemos analizado la forma en la que las facultades públicas españolas presentan las ofertas de Grado en Periodismo a través de los portales web y hemos profundizado en las caracteristicas de los planes de estudios de aquellas materias cuyo contenido responde a las exigencias de narrativas digitales como multimedia, transmedia e hipertexto.
\end{abstract}

Palabras clave: nuevas narrativas, transmedia, gestión de comunicación, periodismo y procesos de enseñanza universitaria 


\section{Introducción y marco teórico}

Una de las principales innovaciones en el periodismo digital en los últimos años son las nuevas narrativas, la búsqueda de nuevas formas de aproximación al lector especialmente la transmedia (Irala, 2014; Alzamora, Tárcia, 2012; Larrondo, 2016). Al mismo tiempo, estas denominadas nuevas narrativas se fueron haciendo un hueco también como estrategia para la enseñanza y el aprendizaje (McEwan; Egan, 1998). Es precisamente en este doble sentido en el que se realiza el análisis propuesto por esta comunicación: por una parte, como elemento a tener en cuenta dentro de la evolución del Periodismo y, por tanto, importante en los estudios sobre el mismo, y, en otro sentido, como herramienta de enseñanza-aprendizaje. Antes de la metodología y el análisis de los resultados se ofrece una aproximación a las nuevas narrativas en periodismo.

Los Laboratorios de Innovación constituyen el elemento visible de una apuesta importante por la experimentación en los medios de comunicación digitales (Salaverría, 2015; Peñafiel, 2016; Bender, 2004; Serrano-Cobos, 2016) fundamentalmente en la búsqueda de nuevas formas de contar historias basadas en hechos reales que atrapen al lector. Los documentos textuales eran inicialmente volcados en la red y en esa búsqueda de nuevas narrativas fueron creados los hipertextos, los documentos multimedia e interactivos adaptándose paulatinamente a las características de la red (Bolter, 1991; Díaz-Noci, 2001).

Siguiendo a Gifreu (2013) y sus estudios sobre el documental, como forma de la no ficción interactiva, podemos definir cuatro grandes fases en la evolución de las narrativas multimedia e interactivas y su convergencia con el periodismo:

1. Aparición (1980-1990). Se corresponde con la emergencia del medio interactivo y los primeros desarrollos experimentales sobre soportes físicos de memoria óptica. Si bien, las narrativas no lineales y transmedia fueron puestas en práctica con anterioridad en los medios analógicos.

2. Experimentación (1990-2000). La popularización del ordenador personal impulsa los productos culturales en soporte CD-ROM y DVD-ROM, mientras que se inicia la presencia de los medios en Internet.

3. Consolidación (2000-2010). La evolución de la red y su expansión permite un desarrollo progresivo de las producciones en línea, sustituyendo los soportes físicos y confluyendo con los medios sociales y los dispositivos móviles.

4. Diversificación (2010-actualidad). El crecimiento de la producción del documental interactivo y el reportaje multimedia definen el escenario actual, donde los medios apuestan por la innovación narrativa e implementan nuevas propuestas, como la realidad virtual, el periodismo de datos y las estrategias transmedia.

El concepto de narrativa carece por el momento de una definición unívoca en las Ciencias Sociales. Desde que Todorov en 1969 acuñó el término narratología para designar la nueva teoría de la narración literaria, la definición de narración evolucionó a través de las que enfocan el concepto en la configuración del mensaje (Smith, 1981), con definiciones que incluyen las nociones de tiempo y propósito (Phela 2005) o las que incluyen al receptor y el consumo de mensajes como parte de la narrativa (Ochs and Capps, 2001; Seale, 2000). A partir de los diferentes modelos de análisis de narrativas de diferentes ámbitos (también el periodístico) se elaboraron modelos de estudio (destacamos los de Propp, 1968; Labov,Waletsky, 1977, Genette, 1972, Ricoeur, 1984) hasta hoy en los que la narrativa se concibe a través de un status que supera al texto para comprenderlo como un fenómeno desarrollado en un determinado contexto comunicativo que en relación directa con él establece un nuevo significado y campo de análisis (Hyvärinen, 2007). En esta comunicación asumimos este enfoque que entiende la narración como una representación

(cc) EY-NC-ND 2017, Universitat Politècnica de València 
de la experiencia construida a través del discurso en el que se otorga sentido, se posibilita su comunicación y se orienta la acción.

Pese a las diferentes conceptualizaciones de estas nuevas fórmulas (crossmedia, multiplataforma, multimodalidad, hiperserialidad, hiperdiégesis, media híbridos, transficción, narrativas transmediales, plataformas múltiples y mercancía intertextual, inmersivo), los estudios recientes sobre narrativas se han fijado sobre todo en la dimensión transmedia.

El término transmedia nace específicamente en un texto titulado Transmedia Storytelling de Jenkins (2003) si bien se indica en varias ocasiones raíces anteriores (Freeman, 2014 o Montoya; Vásquez; Salinas, 2013) e incluso algunos autores observan el uso de diferentes canales para comunicar desde el inicio de los tiempos (Moloney, 2011). Las dificultades conceptuales de la narrativa transmedia en la actualidad (Mora, 2014; Rodríguez Ferrándiz, Peñamarín, 2014) constituyen un verdadero desafío para la narratología y la semiótica textual narrativa ya que como apunta Scolari se trata de:

Campos de estudio fuertemente monomedia - existe una semiótica del teatro, de la radio, del cine, etcétera, pero todavía no tenemos una semiótica del transmedia - y poco acostumbrados al análisis de narrativas multimodales de alta complejidad, caracterizadas por textualidades hiperfragmentadas, y decenas de personajes y programas narrativos (Guerrero, Scolari, 2016).

En este trabajo asumiremos la definición de una estructura narrativa diferenciada que se expande a través de varios medios que cuentan algo diferente, con la participación del usuario y la capacidad del mundo narrativo de ser abordado a través de cualquiera de las unidades textuales que la componen (Jenkins, 2003, 2006; Scolari, 2009). Frente a los conceptos multiplataforma y crossmedia, basados en los soportes y la combinación entre ellos y los usuarios, no son una adaptación de un lenguaje o un mensaje a diferentes soportes (Rodríguez-Ferrándiz; Ortiz; Sáez, 2014) sino creaciones concebidas inicialmente como transmediáticas.

\section{Objetivos y metodología}

Con este estudio queremos mostrar como las universidades públicas españolas presentan la información sobre los grados en periodismo ofertados a través de sus páginas webs y determinar si aprovechan las nuevas narrativas para hacerlo - estudiaremos la estructura y acceso al contenido - . Por otro lado, atendemos a las características de los grados y a las particularidades de su contenido. La recopilación de datos se realizó entre los días 2 y 10 de marzo de 2017 en las páginas web oficiales y en los planes de estudio (atendiendo tanto a la parte teórica como a seminarios y prácticas) correspondientes al curso académico $2016 / 2017$.

El estudio de las web de las facultades parte de la estructura de la página, la distribución del contenido en la misma así como sus características. Para ello determinamos una batería de categorías de análisis distribuidas en de tres partes: contenido multimedia e hipertexto, contenido específico e interactividad y participación.

El primer apartado atiende al uso de imágenes, contenido audiovisual o enlaces que ofrece la página web en su portada. Por otro lado, en el contenido específico detallaremos si el portal incluye información como el plan de estudios, profesorado, salidas profesionales, apartado de noticias (de la propia facultad en la que se imparte el Grado en Periodismo), servicios tales como agenda, contacto...

En el tercer epígrafe estudiamos la posibilidad que tienen los estudiantes interesados para ponerse en contacto con el centro universitario e interactuar; bien en la propia web, a través de un formulario o a través de redes sociales referenciadas en el portal. 
A continuación completamos el estudio de los portales web con un análisis más específico del grado en Periodismo. Partimos de la distribución de créditos en cuanto a cinco ejes básicos: materias de formación básica, materias obligatorias, asignaturas optativas, prácticas externas en empresas y Trabajo Final de Grado. A esto sumamos una categorización de asignatura segundo el medio de comunicación o área base (prensa, radio, televisión, medios digitales, comunicación corporativa...), sobre conocimientos básicos de diferentes ámbitos con influencia en el periodismo (derecho, política, historia, economía) y sobre conocimientos específicos (redacción, locución, géneros, técnicas narrativas...).

A partir de esta categorización, hacemos una selección de aquellas materias relacionadas con el ámbito digital y con las nuevas narrativas emergidas tras la evolución del periodismo hacia el 2.0. Así, a través de los programas docentes — correspondientes a esta última selección- queremos conocer los contenidos y técnicas que ofrecen a quienes cursan dicha materia. Nos centraremos en la especialización que ofrecen así como las características de sus prácticas y seminarios: si atiende al uso de nuevas técnicas de narración como el transmedia, si echa mano de nuevas plataformas social media u otros canales, si su bibliografía va acorde con la rápida evolución de la materia en el ámbito, si se apoya en trabajos dinámicos y de creación o si los trabajos académicos simplemente son teóricos.

Observamos pues interesante abordar esta dimensión que atiende al contenido tanto de páginas web de facultades como de materias e incluirla como una nueva idea, no solo de base para el cambio sino también de innovación, en los procesos de enseñanza-aprendizaje de estudios universitarios.

\section{Presentación de resultados}

\section{1. ¿Cómo presentan la información los portales web de los grados en Periodismo? Información de servicio, sin llamar la atención del público}

La primera toma de contacto de un alumno con un grado universitario probablemente sea a través de la página web oficial de la institución que la oferta. Es necesario por tanto que este portal sea un reflejo de lo que realmente significa el plan de estudios. La primera impresión de la web es esencial así como su funcionalidad. Por este motivo hemos analizado como se presentan los grados de Periodismo en las universidades públicas en España. En una primera aproximación se ha analizado la estructura de éstas y se observó que la mayoría no cuenta con una URL única, por lo que son extensiones de la página oficial de la universidad que alberga el grado. De las analizadas, solo la facultad de Periodismo de la Universidad Miguel Hernández ofrece una web propia con contenidos creados por alumnos entre los que destaca la revista 'Reporteros'. Las demás no hacen referencia a contenidos propios en portada, excepto en contadas ocasiones a través de vídeos que funcionan a modo de incentivo y captación de alumnos como es el caso de la oferta de grado de la Universidad de Navarra. En este sentido, se observa como no hacen uso de las nuevas narrativas y potencialidades de lo digital para 'vender' sus contenidos. Tampoco aprovechan el producto generado por el alumnado como muestra de los resultados en seminarios, por poner un ejemplo.

Si nos paramos en la estructura propiamente dicha, el $60 \%$ de las webs analizadas presenta una distribución a tres columnas. Con respecto al número de 'apartados' en el menú, dos ofrecen una amplia selección con más de 30 'sub-menús', ocho de los portales presentan más de 20, nueve registran más de 10 y solo dos de las web tienen menos de 10 opciones.

Por otro lado se ha observado si la página ofrece la opción de login a la intranet o a la aula virtual desde la portada. Tras el estudio se constata que ocho de las 21 webs objeto de estudio no presentan esta opción desde la pantalla de inicio por lo que dificulta el acceso 'directo' a la plataforma virtual. Sí viene referenciado, sin embargo, la opción de 'Ayuda o contacto' con el centro en caso de solicitar información o solucionar dudas. 
Atendiendo a las características del contenido que ofrece la página observamos que el número de gráficos y audio es nulo, el vídeo solo aparece en cuatro portales web y las imágenes - aunque se usan hasta en 13 ocasiones - solo apoyan el diseño de la web y no buscan llamar al estudiante. Con todo, el uso de enlaces - y por extensión del hipertextoes generalizado, especialmente si atendemos a contenido específico del grado: horarios del curso, tutorías, planes de estudio, docencia...

Otro de los apartados de la ficha de análisis de las portadas de los portales hace hincapié en el contenido específico sobre cursos y profesorado. Constatamos que todas las webs analizadas ofrecen información de las materias. No ocurre lo mismo con la información relativa a los horarios; esta no aparece como apartado único en un $25 \%$ de los casos. La misma tendencia se da en cuanto a la información del profesorado al no aparecer en seis de los portales analizados. Así pues, también se observa que el $40 \%$ de las páginas web no informan sobre las salidas profesionales del grado, pero además, las que si lo hacen no incorporan en sus discursos los nuevos perfiles para los que los periodistas deben estar capacitados en la era 2.0 .

A lo anterior hay que añadir la carencia de actualización e información del día a día que denotan los portales. Solo tres presentan apartado específico de 'Agenda' y la web de la Complutense es la única que añade una sección específica de 'Noticias' relativas a la universidad y al grado en Periodismo en concreto.

En este primer apartado de análisis hay una referencia especial a la participación e interactividad que presentan las portadas de las webs. En un primer lugar se observa que el acceso directo, además de gestión, en redes sociales es del $60 \%$, pero hay que apuntar que solo 4 de estas son específicas para el grado en Periodismo, o para la facultad de Comunicación; el resto responden a la institución universitaria. No sacan partido a los contenidos creados en la propia carrera universitaria y tampoco aprovechan la difusión que las plataformas digitales ofrecen como canales de distribución de contenidos. Dentro de las que si gestionan este recurso/herramienta para llegar a sus estudiantes y a sus potenciales alumnos hacen un uso mayor de Facebook y Youtube, seguido de la red de microblogging Twitter.

En cuanto a la posibilidad de interactuar dentro del propio portal, esta conexión únicamente se materializa a través de formularios. Así, casi el 50\% de las webs analizadas hacen uso de él. Sin embargo, la posibilidad de comentar no es posible, excepto en aquellas webs — seis en total- que disponen de un 'Buzón de sugerencias o quejas', eso sí, no es público y funciona como el formulario. Cabe destacar el servicio ‘¿Te llamamos?', ofertado por la Universidad de Salamanca en el apartado dedicado al grado en Periodismo.

\subsection{Comparativa de los planes de estudios: estructura y distribución de materias}

Con la implementación del grado en lugar de la licenciatura en Periodismo, los planes de estudio responden a períodos formativos compuestos por un total de 240 créditos denominados ETC en cuatro cursos. La categorización de estos responde a tres parámetros básicos: materias obligatorias, de formación básica y optativas. A continuación presentamos esta distribución en los grados analizados así como los itinerarios/especialización que ofertan $-\mathrm{y}$ que aparecen referenciados de forma explícita en los planes de estudio en versión digital-: 
Tabla 1. Distribución de créditos e itinerarios ofertados en grado

\begin{tabular}{|c|c|c|c|c|}
\hline UNIVERSIDAD & $\begin{array}{c}\text { ETC } \\
\text { Obligatoria } \\
\end{array}$ & $\begin{array}{c}\text { ETC } \\
\text { Optativa } \\
\end{array}$ & $\begin{array}{c}\text { ETC } \\
\text { Básica }\end{array}$ & ITINERARIOS \\
\hline $\begin{array}{l}\text { UPF Pompeu } \\
\text { Fabra }\end{array}$ & 104 & 52 & 62 & $\begin{array}{c}4 \text { (Economía, Humanidades, Política, } \\
\text { Derecho) }\end{array}$ \\
\hline UV Valencia & 139,5 & 22,5 & 60 & NO \\
\hline $\begin{array}{l}\text { UNIZAR } \\
\text { Zaragoza }\end{array}$ & 150 & 24 & 60 & NO \\
\hline Carlos III Madrid & 120 & 144 & 60 & $\mathrm{NO}$ \\
\hline $\begin{array}{l}\text { USC Santiago de } \\
\text { Compostela }\end{array}$ & 132 & 60 & 66 & NO \\
\hline $\begin{array}{c}\text { ULL La Laguna } \\
\text { Tenerife }\end{array}$ & 126 & 24 & 60 & NO \\
\hline $\begin{array}{l}\text { UAB Autónoma } \\
\text { de Barcelona }\end{array}$ & 102 & 60 & 60 & $\begin{array}{c}4 \text { (Ciberperiodismo, Análisis/Planificación, } \\
\text { Política/econ, Cultura/sociedad) }\end{array}$ \\
\hline Jaume I Castellón & 138 & 45 & 66 & NO \\
\hline UM Murcia & 144 & 18 & 60 & $\mathrm{NO}$ \\
\hline $\begin{array}{l}\text { Rey Juan Carlos } \\
\text { Madrid }\end{array}$ & 144 & 18 & 60 & NO \\
\hline UMA Málaga & 120 & 54 & 60 & NO \\
\hline US Sevilla & 120 & 54 & 60 & $\mathrm{NO}$ \\
\hline $\begin{array}{l}\text { UMH Miguel } \\
\text { Hernández Elche }\end{array}$ & 150 & 24 & 60 & NO \\
\hline $\begin{array}{c}\text { UCM } \\
\text { Complutense } \\
\text { Madrid }\end{array}$ & 108 & 66 & 60 & NO \\
\hline EHU País Vasco & 120 & 48 & 60 & $\begin{array}{c}1 \text { (Mención en Dirección de } \\
\text { Comunicación) }\end{array}$ \\
\hline $\begin{array}{l}\text { UCLM Castilla } \\
\text { La Mancha }\end{array}$ & 120 & 54 & 60 & $\begin{array}{c}2 \text { (Periodismo Digital y Periodismo } \\
\text { Especializado) }\end{array}$ \\
\hline UVA Valladolid & 126 & 30 & 60 & NO \\
\hline UNAV Navarra & 141 & 30 & 60 & $\begin{array}{l}2 \text { (Comunicación Institucional y } \\
\text { Comunicación en Moda) }\end{array}$ \\
\hline UPSA Salamanca & 168 & 6 & 60 & NO \\
\hline $\begin{array}{l}\text { URV Rovira i } \\
\text { Virgili }\end{array}$ & 120 & 36 & 60 & NO \\
\hline Illas Baleares & 126 & 24 & 60 & NO \\
\hline
\end{tabular}

Con respecto a los ETC de TFG y Prácticas Externas, el número varía y se encuentra en el primer caso entre los 6 y los 12 créditos y en el trabajo final entre los 6, 12, 18 o incluso 24 en el grado en la Rey Juan Carlos.

Profundizando en las características de las materias ofertadas, se procede a una clasificación en cuanto a especialidad por canal: radio, televisión (audiovisual), prensa y ciberperiodismo o periodismo digital. A estas tres, se ha añadido la comunicación corporativa por ser ésta y los gabinetes, en general, una área importante de la comunicación y especialmente un campo que recibe un gran número de titulados en Periodismo especialmente en la era 2.0 con la necesidad de gestionar cuentas oficiales en plataformas digitales como las redes sociales, por ejemplo-. 
Tabla 2. Distribución de asignaturas por especialidad en cuanto a canal y formación

\begin{tabular}{|c|c|c|c|c|c|c|c|}
\hline UNIVERSIDAD & DIG. & RADIO & TV & PR. & $\begin{array}{l}\text { COM. } \\
\text { CORPOR. }\end{array}$ & $\begin{array}{c}\text { ESP. } \\
\text { DESTREZA }\end{array}$ & F. CONOC. \\
\hline UPF Pompeu Fabra & 1 & $\mathrm{X}$ & 1 & $\mathrm{X}$ & $\mathrm{X}$ & 29 & 84 \\
\hline UV Valencia & 1 & 1 & 1 & $\mathrm{X}$ & 1 & 17 & 20 \\
\hline UNIZAR Zaragoza & 3 & 4 & 6 & 1 & 2 & 26 & 15 \\
\hline Carlos III Madrid & 3 & 1 & 3 & $\mathrm{X}$ & 1 & 25 & 20 \\
\hline $\begin{array}{l}\text { USC Santiago de } \\
\text { Compostela }\end{array}$ & 4 & 1 & 4 & 2 & 2 & 14 & 16 \\
\hline $\begin{array}{l}\text { ULL La Laguna } \\
\text { Tenerife }\end{array}$ & 1 & 1 & 4 & 1 & 1 & 11 & 21 \\
\hline $\begin{array}{l}\text { UAB Autónoma de } \\
\text { Barcelona }\end{array}$ & 4 & 1 & 4 & 2 & 2 & 28 & 23 \\
\hline Jaume I Castellón & 1 & 3 & 5 & $\mathrm{X}$ & $\mathrm{X}$ & 20 & 16 \\
\hline UM Murcia & 1 & 1 & 1 & $\mathrm{X}$ & $\mathrm{X}$ & 19 & 16 \\
\hline $\begin{array}{l}\text { Rey Juan Carlos } \\
\text { Madrid }\end{array}$ & 2 & 2 & 4 & 1 & $X$ & 11 & 15 \\
\hline UMA Málaga & 2 & 2 & 5 & 2 & 1 & 19 & 21 \\
\hline US Sevilla & $\mathrm{X}$ & $\mathrm{X}$ & 4 & 1 & 1 & 26 & 37 \\
\hline $\begin{array}{c}\text { UMH Miguel } \\
\text { Hernández Elche }\end{array}$ & 1 & 1 & 4 & $\mathrm{X}$ & 1 & 19 & 16 \\
\hline $\begin{array}{c}\text { UCM Complutense } \\
\text { Madrid }\end{array}$ & 1 & 1 & 2 & 2 & 1 & 15 & 37 \\
\hline EHU País Vasco & 2 & 1 & $\mathrm{X}$ & 1 & 3 & 11 & 8 \\
\hline $\begin{array}{c}\text { UCLM Castilla La } \\
\text { Mancha }\end{array}$ & 4 & 2 & 2 & $X$ & 1 & 20 & 13 \\
\hline UVA Valladolid & 2 & 1 & 3 & $\mathrm{X}$ & 1 & 18 & 16 \\
\hline UNAV Navarra & 2 & 3 & 3 & 1 & $\mathrm{X}$ & 9 & 26 \\
\hline UPSA Salamanca & 1 & 2 & 3 & 1 & 1 & 12 & 27 \\
\hline $\begin{array}{l}\text { URV Rovira i } \\
\text { Virgili }\end{array}$ & 2 & $X$ & 1 & $X$ & 1 & 6 & 16 \\
\hline Illas Baleares & 2 & 2 & 3 & 1 & 2 & 7 & 11 \\
\hline
\end{tabular}

Con el estudio, en el que se han tenido en cuenta tanto las asignaturas optativas como las que conforman bloques concretos de especialización, se ha detectado un mayor número de materias centradas en lo audiovisual. En segundo lugar están las relativas al mundo digital pero con un carácter práctico de edición como veremos más adelante al hablar del contenido y del tratamiento de nuevas narrativas. En cuanto a la radio y prensa, éstas se sitúan en tercer y cuarto lugar. Incluso las materias relacionadas con la comunicación en gabinetes/organizacional acumulan un mayor número de horas - aunque hablemos en este caso de materias optativas, por lo general - que las que atienden a contenidos para medios impresos. 
Además de esta clasificación, el estudio incorpora otra diferenciación para materias cuya base es la formación de capacidades para el desarrollo del rol de periodista - especializadas en destrezas propias de la profesión-y por otro lado para aquellas que, aunque forman al periodista en temas que lo involucran y de los que debe estar al tanto, son más de formación básica: economía, historia (del periodismo, de España, de los cibermedios...).

Atendiendo por tanto a esta clasificación se ha detectado un gran número de materias sobre otros campos del conocimiento que aportan el bagaje necesario del periodista. Esta tendencia se da incluso en las materias optativas. De las universidades analizadas, la Pompeu Fabra es la que se posiciona en primer lugar con un total de 84 materias, seguida de los grados en la Complutense y en la Universidad de Sevilla con 37 asignaturas cada una. Con todo, la media está en 23 materias con este carácter.

\subsection{El tratamiento y estudio de nuevas narrativas en las materias especializadas en el ámbito digital}

La base de esta investigación es el análisis de los planes de estudio de las materias enmarcadas en el ámbito digital. Así pues, se han seleccionado aquellas que, de alguna forma, versan sobre el tratamiento informativo o forman al alumnado en destrezas para el ciberperiodismo. Con el fin de determinar las características de los contenidos que presentan, se elabora una ficha basada en un método cualitativo y que atiende a dos vertientes: por un lado la teórica y por otro la práctica (seminarios y clases interactivas de laboratorio). Dentro de estos dos ejes, se han estudiado diferentes categorías que se presentan a continuación con los resultados obtenidos.

\subsubsection{De la especialización en soportes a las exigencias de los nuevos lenguajes narrativos}

Lo de hacer periodismo online requiere el conocimiento previo de las exigencias del canal y por tanto también de la forma en la que vamos a expresar la idea que se quiere transmitir. Es por ello que la irrupción del mundo digital obliga a establecer cambios que derivan en nuevas narrativas, que deben ser una base fundamental en los procesos enseñanzaaprendizaje.

En los 21 grados analizados, seleccionamos 44 materias como aquellas que versan sobre aspectos del periodismo digital. Es decir, hablamos de dos materias aproximadamente por grado - entre las que la mayoría son de carácter optativo-, un número muy bajo teniendo en cuenta las exigencias de la actualidad.

En este estudio se ha observado que 38 de las 44 materias analizadas enfocan sus contenidos a la creación, edición y producción de noticias para portales web. De éstas, un $45 \%$ también dedica algún tema a la gestión de redes sociales así como a su presentación como nuevos canales en los que distribuir contenido. Cabe señalar que además de estos contenidos, el funcionamiento de las bases de datos y su utilidad forman parte de los temas del plan de estudios de tres asignaturas analizadas: 'Nuevos Soportes y Arquitectura de la Información' (USC), 'Construcción de Servicios de Información Digital' (UM) y 'Ciberperiodismo' (UNAV).

Además de lo anterior, hay que destacar la forma en que tratan la información para los canales señalados. Emergen aquí los conceptos hipertexto, hipermedia, multimedia o interactivo para referirse a las narrativas que acaparan las materias más recientes en los estudios universitarios de Periodismo. Pero, ¿abarcan estos términos las múltiples posibilidades narrativas que han surgido y que propician las plataformas 2.0 en el discurso periodístico? Tras el estudio podemos afirmar que los programas de las materias distan mucho de lo que se está haciendo en realidad. De hecho, la investigación reserva un apartado especial al estudio de las narrativas transmedia, y solo se detectó su presencia en 12 de las 38 materias seleccionadas. Es más, esta presencia se reduce a una referencia 
bibliográfica en 5 materias siendo el resto parte del temario y, en el mejor de los casos, entendido como estrategia editorial ('Nuevos Formatos y Productos para la Red', USC) o como fenómeno fan, especialmente en contenidos de ficción ('Comunicación y Participación Ciudadana en la Red', Carlos III Madrid).

Tabla 3. Distribución de materias según narrativas e información sobre bibliografía

\begin{tabular}{|c|c|c|c|c|}
\hline UNIVERSIDAD & ASIGNATURA & $\begin{array}{c}\text { TIPO DE } \\
\text { NARRATIVA }\end{array}$ & $\begin{array}{c}\text { ESP. } \\
\text { TRANSMEDIA }\end{array}$ & BIBLIOGRAFÍA \\
\hline $\begin{array}{l}\text { UPF Pompeu } \\
\text { Fabra }\end{array}$ & $\begin{array}{c}\text { Análisis del Periodismo } \\
\text { Digital }\end{array}$ & NO & $\mathrm{NO}$ & $2009 \_2014$ \\
\hline UV Valencia & Periodismo Digital & $\mathrm{NO}$ & $\mathrm{NO}$ & 2001_2016 \\
\hline \multirow[t]{3}{*}{$\begin{array}{l}\text { UNIZAR } \\
\text { Zaragoza }\end{array}$} & $\begin{array}{l}\text { Comunicación e } \\
\text { Información Digital }\end{array}$ & $\begin{array}{l}\text { Hipertexto, } \\
\text { multimedia }\end{array}$ & NO & 2004_2012 \\
\hline & $\begin{array}{c}\text { Proyecto de } \\
\text { Comunicación Digital }\end{array}$ & $\begin{array}{l}\text { Hipertexto, } \\
\text { multimedia }\end{array}$ & NO & $2005 \_2012$ \\
\hline & $\begin{array}{c}\text { Narrativa Audiovisual: } \\
\text { Reportaje y } \\
\text { Documental }\end{array}$ & NO & NO & $1999 \_2011$ \\
\hline \multirow[t]{4}{*}{ Carlos III Madrid } & Periodismo en Red & $\begin{array}{l}\text { Hipertexto, } \\
\text { multimedia }\end{array}$ & NO & 1995_2014 \\
\hline & $\begin{array}{l}\text { Comunicación y } \\
\text { Participación } \\
\text { Ciudadana en Red }\end{array}$ & No especifica & $\begin{array}{l}\text { Transmedia } \\
\text { como fenómeno } \\
\text { fan }\end{array}$ & 1997_2014 \\
\hline & $\begin{array}{c}\text { Comunicación } \\
\text { Multimedia: el relato } \\
\text { periodístico }\end{array}$ & $\begin{array}{l}\text { Hipertexto, } \\
\text { multimedia } \\
\text { (Apartado } \\
\text { específico narr. } \\
\text { interactivas) }\end{array}$ & NO & 2012 \\
\hline & $\begin{array}{l}\text { Recursos Narrativos } \\
\text { para el Relato } \\
\text { Periodístico }\end{array}$ & $\begin{array}{l}\text { La micronoticia. } \\
\text { Twitter }\end{array}$ & NO & No especifica \\
\hline \multirow[t]{4}{*}{$\begin{array}{l}\text { USC Santiago de } \\
\text { Compostela }\end{array}$} & Multimedia & $\begin{array}{l}\text { Hipertexto, } \\
\text { multimedia }\end{array}$ & $\begin{array}{c}\text { Solo en } \\
\text { bibliografía }\end{array}$ & 2001_2015 \\
\hline & $\begin{array}{l}\text { Teoría y Análisis de } \\
\text { Cibermedios }\end{array}$ & Hipert, multime & NO & 2001_2016 \\
\hline & $\begin{array}{l}\text { Nuevos Soportes y } \\
\text { Arquitectura de la } \\
\text { Información }\end{array}$ & Varias & $\begin{array}{c}\text { Tema } \\
\text { específico }\end{array}$ & 1999_2016 \\
\hline & $\begin{array}{l}\text { Nuevos Formatos y } \\
\text { Productos para la Red }\end{array}$ & $\begin{array}{l}\text { Hipertexto, } \\
\text { multimedia }\end{array}$ & $\begin{array}{c}\text { Transmedia } \\
\text { como estrategia } \\
\text { editorial }\end{array}$ & $2000 \_2010$ \\
\hline $\begin{array}{l}\text { ULL La Laguna } \\
\text { Tenerife }\end{array}$ & Ciberperiodismo & $\begin{array}{l}\text { Hipertexto, } \\
\text { multimedia }\end{array}$ & $\mathrm{NO}$ & 2001_2016 \\
\hline \multirow[t]{2}{*}{$\begin{array}{l}\text { UAB Autónoma } \\
\text { de Barcelona }\end{array}$} & $\begin{array}{l}\text { Escritura Periodística } \\
\text { en Multimedia e } \\
\text { Interactivos }\end{array}$ & $\begin{array}{l}\text { Hipertexto, } \\
\text { multimedia }\end{array}$ & $\begin{array}{c}\text { Solo en } \\
\text { bibliografia }\end{array}$ & 1995_2015 \\
\hline & Géneros y Formatos & Hipertexto, & Tema & $2003 \_2015$ \\
\hline
\end{tabular}




\begin{tabular}{|c|c|c|c|c|}
\hline & $\begin{array}{l}\text { Audiovisuales } \\
\text { Multimedia }\end{array}$ & $\begin{array}{l}\text { multimedia, } \\
\text { transmedia }\end{array}$ & específico & \\
\hline & $\begin{array}{c}\text { Producción Periodística } \\
\text { Multiplataforma }\end{array}$ & $\begin{array}{l}\text { Hipertexto, } \\
\text { multimedia }\end{array}$ & $\begin{array}{c}\text { Solo en } \\
\text { bibliografía }\end{array}$ & 2000_2012 \\
\hline & $\begin{array}{c}\text { Tendencias de } \\
\text { Desarrollo de Internet }\end{array}$ & NO & NO & 1990_2016 \\
\hline & $\begin{array}{c}\text { Gestión de Contenidos } \\
\text { Digitales }\end{array}$ & No especifica & NO & 1999_2011 \\
\hline \multirow[t]{2}{*}{$\begin{array}{l}\text { Jaume I } \\
\text { Castellón }\end{array}$} & Ciberperiodismo & No especifica & $\begin{array}{c}\text { Solo en } \\
\text { bibliografía }\end{array}$ & 2000_2015 \\
\hline & Narración audiovisual & Audiovisuales & NO & 1991_2011 \\
\hline UM Murcia & $\begin{array}{l}\text { Construcción de } \\
\text { Servicios de } \\
\text { Información Digital }\end{array}$ & No especifica & NO & 1998_2012 \\
\hline \multirow[t]{2}{*}{$\begin{array}{l}\text { Rey Juan Carlos } \\
\text { Madrid }\end{array}$} & $\begin{array}{c}\text { Planificación y } \\
\text { desarrollo de proyectos } \\
\text { en la red }\end{array}$ & $\begin{array}{l}\text { Hipertexto, } \\
\text { multimedia }\end{array}$ & $\begin{array}{c}\text { Análisis } \\
\text { desarrollo de } \\
\text { narr. transmedia } \\
\text { globales }\end{array}$ & 2001_2016 \\
\hline & Periodismo multimedia & $\begin{array}{l}\text { Hipertexto, } \\
\text { multimedia }\end{array}$ & NO & 1998_2015 \\
\hline \multirow[t]{2}{*}{ UMA Málaga } & Información en Internet & $\begin{array}{l}\text { Hipertexto, } \\
\text { multimedia }\end{array}$ & NO & 2002_2013 \\
\hline & $\begin{array}{l}\text { Periodismo Ciudadano } \\
\text { y Redes Sociales }\end{array}$ & $\begin{array}{l}\text { Hipertexto, } \\
\text { multimedia e } \\
\text { interactivas }\end{array}$ & NO & 2003_2012 \\
\hline US Sevilla & No hay materias & - & - & - \\
\hline $\begin{array}{l}\text { UMH Miguel } \\
\text { Hernández Elche }\end{array}$ & $\begin{array}{c}\text { Comunicación Digital e } \\
\text { Internet }\end{array}$ & No especifica & NO & 1994_2010 \\
\hline $\begin{array}{c}\text { UCM } \\
\text { Complutense } \\
\text { Madrid }\end{array}$ & Multimedia & $\begin{array}{l}\text { Hipermedia, } \\
\text { multimedia e } \\
\text { interactivas }\end{array}$ & $\begin{array}{c}\text { Tema } \\
\text { específico }\end{array}$ & 2002_2010 \\
\hline \multirow[t]{2}{*}{ EHU País Vasco } & $\begin{array}{l}\text { Edición y Produción } \\
\text { Multimedia }\end{array}$ & $\begin{array}{l}\text { Hipertexto, } \\
\text { multimedia }\end{array}$ & $\begin{array}{c}\text { Solo en } \\
\text { bibliografía }\end{array}$ & 2000_2015 \\
\hline & $\begin{array}{l}\text { Periodismo Social en } \\
\text { Internet }\end{array}$ & $\begin{array}{l}\text { Narrativas en } \\
\text { storify }\end{array}$ & NO & 2002_2011 \\
\hline \multirow[t]{4}{*}{$\begin{array}{l}\text { UCLM Castilla } \\
\text { La Mancha }\end{array}$} & $\begin{array}{l}\text { Técnicas del Mensaje } \\
\text { en Prensa e Internet }\end{array}$ & No especifica & NO & 1999_2009 \\
\hline & Ciberperiodismo & No especifica & NO & 2011_2015 \\
\hline & $\begin{array}{l}\text { Taller de Periodismo } \\
\text { Multimedia I }\end{array}$ & No especifica & NO & 2000_2013 \\
\hline & $\begin{array}{l}\text { Taller de Periodismo } \\
\text { Multimedia II }\end{array}$ & No especifica & NO & 2000_2013 \\
\hline UVA Valladolid & Ciberperiodismo & No especifica & $\begin{array}{c}\text { Solo en } \\
\text { bibliografía }\end{array}$ & 2001_2016 \\
\hline
\end{tabular}




\begin{tabular}{|c|c|c|c|c|}
\hline & $\begin{array}{c}\text { Periodismo } \\
\text { Participativo en la Red }\end{array}$ & En red & NO & 2000_2012 \\
\hline \multirow[t]{2}{*}{ UNAV Navarra } & $\begin{array}{l}\text { Comunicación } \\
\text { Multimedia }\end{array}$ & $\begin{array}{l}\text { Hipertexto, } \\
\text { multimedia e } \\
\text { interactivas }\end{array}$ & NO & 2008_2015 \\
\hline & Ciberperiodismo & $\begin{array}{l}\text { Hipermedia, } \\
\text { multimedia e } \\
\text { interactivas }\end{array}$ & $\begin{array}{l}\text { NO (Aunque } \\
\text { habla de } \\
\text { formatos } \\
\text { integrados) }\end{array}$ & 2003_2017 \\
\hline $\begin{array}{c}\text { UPSA } \\
\text { Salamanca }\end{array}$ & Periodismo Digital & NO & NO & 2003_2015 \\
\hline \multirow[t]{2}{*}{$\begin{array}{l}\text { URV Rovira i } \\
\text { Virgili }\end{array}$} & $\begin{array}{l}\text { Diseño Gráfico y } \\
\text { Multimedia }\end{array}$ & $\begin{array}{l}\text { Hipertexto, } \\
\text { multimedia }\end{array}$ & NO & 2002_2014 \\
\hline & Periodismo en Internet & $\begin{array}{l}\text { Hipertexto, } \\
\text { multimedia }\end{array}$ & NO & 2005_2014 \\
\hline Illas Baleares & Periodismo Digital & $\begin{array}{l}\text { Hipertexto, } \\
\text { multimedia }\end{array}$ & NO & 2004_2010 \\
\hline
\end{tabular}

\subsubsection{Los seminarios: actualizados en herramientas pero con base narrativa tradicional}

Los planes de estudio de las asignaturas analizadas presentan, por lo general, pocos detalles de la planificación de la materia. No especifican más allá de los índices de los temas si observamos las referencias relativas a la parte teórica de la asignatura. Con todo, los seminarios, aportan mayor información pero esta sigue siendo escueta y no se puede descubrir si realmente llevan un discurso actualizado, si se valen de nuevas herramientas o si sacan partido a las nuevas narrativas no solo como contenido, sino como forma de enseñanza.

La idea de transmedialidad que podría desarrollarse en los procesos de aprendizaje no aparece referenciada en ninguno de los planes estudiados. Incluso la parte teórica de los mismos está 'desconectada' de los seminarios y en muy pocos casos la teoría es llevada a la práctica de forma creativa. Aunque en todos los programas se combinan las clases teóricas con seminarios y prácticas de laboratorio, estas últimas no van más allá de la producción de contenidos lineales que no trascienden del ámbito universitario. El sistema parece que sigue dando prioridad a la parte teórica, dejando para las prácticas externas en empresas el conocimiento real de la evolución del sector periodístico.

En la mayoría de las materias analizadas, el trabajo práctico de seminario consiste en crear un proyecto de carácter multimedia, trabajar en su constante actualización y gestionar redes sociales que aporten valor añadido al mismo. Este se complementa en ciertos casos con herramientas actuales e innovadoras que favorecen la irrupción de nuevos géneros como el multimedia o la webzine que propone la materia 'Información para Internet' en la universidad de Málaga o el Storify en 'Periodismo Ciudadano y Redes Sociales' del mismo programa de grado. Otras como 'Comunicación Digital e Internet' en la Jaume I orientan su discurso hacia el marketing y enfocan su programa hacia la comunicación corporativa, valiéndose además de recursos para desarrollo de contenido en plataformas de social media.

Durante los seminarios son muchos los programas utilizados según muestra el análisis de los planes de estudio. Entre ellos los de edición son los más usados, pero los programas incorporan nuevas herramientas por lo que en este aspecto si que proporcionan a los alumnos recursos actuales. Destacan por tanto programas de análisis en redes sociales como 
Gephy del que hace referencia la materia 'Nuevos Formatos y Productos para la Red' (USC). Sin embargo, detectamos la carencia de seminarios dedicados en exclusiva a técnicas para la aplicación de nuevas narrativas, narrativas que aparecen referenciadas en la bibliografía básica en pocas ocasiones — referencias que están desactualizadas, en los programas aparecen muy pocas de los últimos dos años 2015-2016) -

Una de las dimensiones donde mayor uso tienen las herramientas antes señaladas es en los análisis de caso propuestos en los seminarios. Con todo, observamos que la formación en investigación se reduce a pequeños estudios.

\subsection{Contenidos para la participación. La interacción como finalidad en los procesos digitales}

Una de las características que marcan las asignaturas seleccionadas es la importancia que dan a la gestión de la interacción entre periodista y usuario. En este aspecto se observa una evolución en los planes de estudio ya que en materias donde la radio, la televisión o la prensa son los principales canales, no se atiende a esta nueva dimensión que emerge en la era 2.0. Es tanta la importancia que recibe que incluso hay asignaturas específicas del tema con títulos como 'Comunicación y participación ciudadana en red' en el grado de la Carlos III o 'Periodismo Participativo en la Red' impartida en la Universidad de Valladolid. También se han detectado seminarios y partes teóricas enfocadas a la formación del periodista como community manager. A pesar de que el número de materias para el ámbito digital es muy bajo, si que hay constancia del cambio de los discursos a juzgar por la incorporación de materias como 'Técnicas del Mensaje en Prensa e Internet' que combinan estrategias para ambos canales y ofrecen comparativas.

\section{Conclusiones}

El número de usuarios que cada día accede a webs o tiene aplicaciones móviles con el fin de informarse aumenta día tras día, por ello los contenidos deben ir adaptándose a la forma de consumo de los lectores.

Los planes de estudio de los grados en Periodismo ofertan un número reducido de asignaturas de especialización en estrategias digitales. A esto hay que añadir el carácter optativo de las mismas, cuando las plataformas digitales son los canales actuales de distribución de contenidos. Los planes de estudio deben actualizar sus contenidos y determinar nuevas estrategias, incluyendo materias sobre nuevas narrativas y lenguajes para dar respuesta a las exigencias de los nuevos soportes. Además, los programas deben tener en cuenta la constante evolución de lo digital, por ello la parte teórica debe incorporar resultados de investigaciones recientes que consten como parte de la bibliografía —en esta investigación hay programas donde la referencia más actual data de 2010 -

A lo anterior hay que sumar la necesidad de formación para gestionar la interacción con el lector. Los ciudadanos hoy en día son parte activa de la información gracias a la variedad de herramientas que están a su disposición y en las que dejan constancia de sus opiniones. Por ello, es necesario formar gestores de comunicación, que sepan empatizar con el lector - entendido ahora como participativo e incluso prosumidor- para que el discurso informativo refleje mejor que nunca las ideas latentes en la sociedad. Al fin y al cabo, el periodismo sigue siendo el motor que da visibilidad a la opinión pública por lo que debemos aprovechar el mejor acceso al pensamiento ciudadano. 


\section{Referencias bibliográficas y recursos electrónicos}

ALZAMORA, G. y TÁRCIA, L. (2012). "Convergence and transmedia: semantic galaxies and emergent narratives in journalism" en Brazilian journalism research, vol. 8, 1, p. 22-34. $<$ http://bjr.sbpjor.org.br/bjr/article/view/407/0> [Consulta: 15 de enero de 2017]

BENDER, W. (2004). "The seven secrets of the media lab" en BT technology journal, vol. 22, 4, p. 56.

BOLTER, J. D. (1991). Writing Space. The Computer, Hypertext, and the History of Writing. Nueva Jersey: Lawrence Erlbaum Associates. $<$ https://case.edu/affil/sce/authorship/bolter.pdf $>$ [Consulta: 15 de enero de 2017]

DÍAZ-NOCI, J. (2001). La escritura digital: hipertexto y construcción del discurso informativo en el periodismo electrónico. Bilbao: Universidad del País Vasco. ISBN: 84-8373-378-1 DOI: 10.7764/cdi.38.760

FREEMAN, M. (2014). “Advertising the Yellow Brick Road: Historicizing the Industrial Emergence of Transmedia Storytelling” en International Journal of Communication, 8, p. 2362-2381.

GENETTE, G. (1972). Figures III. Paris: Éditions du Seuil. ISNB: ISBN 978202002039.

GIFREU, A. (2013). El documental interactivo. Evolución, caracterización y perspectivas de desarrollo. Barcelona: Editorial UOC.

GUERRERO-PICO, M. Y SCOLARI, C. (2016). "Narrativas transmedia y contenidos generados por los usuarios: el caso de los crossovers" en Cuadernos.info, 8, 183-200.

HYVÄRINEN, M. (2008). "Analyzing narratives and story-telling” en ALASUUTARI, P.; BICKMAN, L. y BRANNEN, J. The SAGE handbook of social research methods. Sage, p. 447-460.

IRALA HORTAL, P. (2014): "Nuevas narrativas en el periodismo actual. El periodismo transmediático" en Estudios sobre el Mensaje Periodístico. vol. 20, 1, p. 147-158. Madrid, Servicio de $\begin{array}{llll}\text { Publicaciones de la } & \text { Universidad Complutense. }\end{array}$ $<$ http://search.proquest.com/openview/5bf8c2fd2810f17ca1b98f958cfce5b8/1?pqorigsite $=$ gscholar $\&$ cbl $=237533>$ [Consulta: 19 de febrero de 2017]

JENKINS, H. (2003). "Transmedia storytelling. Moving characters from books to films to video games can make them stronger and more compelling” en Technology Review, 10.

JENKINS, H. (2006). Convergence Culture: Where Old and New Collide. New York: University Press. ISBN: 9780814742952

LABOV, W. y WALETSKY, J. (1967). "Narrative analysis: oral versions of personal experience" en Journal of Narrative and Life History, vol. 7, 1-4, p. 3-38.

LARRONDO URETA, A. (2016). "El relato transmedia y su significación en el periodismo. Una aproximación conceptual y práctica" en Trípodos, 38, 31-47.

MCEWAN, H. Y EGAN, K. (comp) (1998). La narrativa en la enseñanza, el aprendizaje y la investigación. Buenos Aires: Amorrortu

MOLONEY, K. (2011). Porting Transmedia Storytelling To Journalism. Denver. $<\mathrm{http}$ //www.colorado.edu/journalism/photojournalism/Transmedia_Journalism.pdf $>$ [Consulta: $15 \mathrm{de}$ enero de 2017] 
MONTOYA, D. F.; VÁSQUEZ, M. Y SALINAS, H. (2013). "Sistemas intertextuales transmedia: exploraciones conceptuales y aproximaciones investigativas" en Revista Coherencia, vol. 10, 18, $\mathrm{p}$ 137-159. <http://publicaciones.eafit.edu.co/index.php/co-herencia/article/view/2136/2093> [Consulta: 19 de marzo de 2017]

MORA, V. L. (2014). “Acercamiento al problema terminológico de la narratividad transmedia" en Caracteres. Estudios culturales y críticos de la esfera digital, vol. 3, 1. $<$ http://revistacaracteres.net/revista/vol3n1mayo2014/problema-terminologico-transmedia/> [Consulta: 19 de marzo de 2017]

OCHS, E. Y CAPPS, L. (2001). Living Narrative. Creating Lives in Everyday Storytelling. Cambridge, MA: Harvard University Press. ISBN: 978-0674010109

PEÑAFIEL, C. (2016). "Reinvención del periodismo en el ecosistema digital y narrativas transmedia" en AdComunica, Revista Científica de Estrategias, Tendencias e Innovación en Comunicación, 12, p. 163-183.

PHELAN, J. (2005). Living to Tell about It. A Rhetoric and Ethics of Character Narration. Ithaca: Cornell University Press. ISBN: 978-0801442971

PROPP, V. (1958). Morphology of the Folktale. Indiana Univ. Research Center in Anthropology. Folklore and Linguistics, 10

RICEUR, P. (1983). Temps et récit I. Paris: Éditions du Seuil. ISBN: 978-2020134521.

RODRÍGUEZ FERRÁNDIZ, R. Y PEÑAMARÍN, C. (2014). "Narraciones transmedia y construcción de los asuntos públicos" en CIC Cuadernos de Información y Comunicación, vol. 19, p. $9-16$

SALAVERRÍA, R. (2015). "Los labs como fórmula de innovación en los medios" en El profesional de la información, vol. 24, 4, p. 397-404. <http://dx.doi.org/10.3145/epi.2015.jul.06> [Consulta: 25 de marzo de 2017]

SCOLARI, C. (2009). "Transmedia Storytelling: Implicit Consumers, Narrative Worlds, and Branding in Contemporary Media Production" en International Journal of Communication, v. 3, p. 586-606.

SEALE, C. (2000). "Resurrective practice and narrative" en ANDREWS, M.; SCLATER, S. D.; SQUIRE, C. y TREACHER, A. Lines of Narrative. Psychosocial Perspectives. London and New York: Routledge. ISBN: 978-0415242332.

SERRANO-COBOS, J. (2016). "Tendencias tecnológicas en internet: hacia un cambio de paradigma" en El profesional de la información, vol. 25, 6, p. 843-850. <https://doi.org/10.3145/epi.2016.nov.01> [Consulta: 2 de enero de 2017]

SMITH, B. H. (1981). "Narrative version, and narrative theories" en MITCHELL, W. J. On Narrative. Chicago: University of Chicago Press. ISBN: 0226532178.

El texto de esta comunicación está elaborado en el marco del proyecto "Usos y preferencias informativas en el nuevo mapa de medios en España: modelos de periodismo para dispositivos móviles" (Referencia: CSO2015-64662-C4-4-R), del Ministerio de Economía y Competitividad. Este proyecto está cofinanciado por el fondo estructural FEDER. 